\title{
Full wave optical profilometry
}

\author{
S. Arhab, G. Soriano, ${ }^{*}$ K. Belkebir, A. Sentenac, and H. Giovannini \\ Institut Fresnel (CNRS UMR 6133), Aix-Marseille Université, Campus de St Jérôme, 13013 Marseille, France \\ *Corresponding author: gabriel.soriano@fresnel.fr
}

Received December 15, 2010; revised January 31, 2011; accepted January 31, 2011; posted February 1, 2011 (Doc. ID 139312); published March 15, 2011

\begin{abstract}
We show that tomographic diffractive microscopy can be used for profilometry applications with high transverse resolution. We present an iterative reconstruction procedure, based on a rigorous wave scattering model, that permits us to retrieve the profile of rough metallic interfaces from the complex scattered field. The transversal resolution is subwavelength, and can even fall below the classical resolution limit if the profile is rough enough for multiple interactions to occur. Large profiles, with tens of wavelength size, can be investigated. (C) 2011 Optical Society of America OCIS codes: $\quad 290.3200,290.5880,050.1755,180.1655$.
\end{abstract}

\section{INTRODUCTION}

Optical profilometry is a nondestructive and noncontact surface metrology technique. Different schemes have been proposed for determining the surface topography of the samples. In those based on a Mirau, Linnik, or Michelson interferometer, the beam reflected by the surface is superimposed to a reference beam. The height variation of the surface profile is determined by processing the signal produced by the resulting interference phenomenon usually observed in white light [1]. In confocal microscopes, a focused beam reflected by the surface is sent to a photodetector placed behind a pinhole. The signal depends on the position along the optical axis of the surface with respect to the focal plane of the microscope objective [2,3]. Scanning the spot laterally gives the topography of the surface. Moving the sample or the microscope objective along the optical axis permits one to increase the vertical dynamic range of measurement. An alternative is given by confocal chromatic sensors. In this case, the light emitted by a broadband source is focused on the surface through a chromatic lens [ㄴ,5]. Analyzing the spectrum of the light passing through the confocal pinhole permits one to determine the profile of the surfaces with no need of vertical scanning. All these techniques assume that the beam reflected by the surface is parallel to the incident one. For this reason, their application is restricted to the case of surfaces with small slopes. Vertical resolution can be of the order of several angstroms, while lateral resolution is limited by diffraction and is not better than $0.5 \mu \mathrm{m}$. Now, with the improvements of nanofabrication, especially in the microelectronic domain, there is a strong need for optical instruments able to retrieve the roughness of surfaces with a typical transverse dimension of about $100 \mathrm{~nm}$. Recent advances in digital tomographic microscopy [6,7] have shown that this quantitative metrology technique can be very powerful for imaging three-dimensional objects. Thanks to the synthetic high numerical aperture obtained by varying the incidence angle, the lateral resolution obtained with this technique is at least twice better than that obtained with classical microscopes. It has also been shown theoretically and experimentally that the resolution can be improved further in the presence of multiple scattering [요, $\underline{9}$ ]. In this case, high spatial frequency components of the field diffracted by the sample that are not detected in the single scattering regime are recorded by the detector. Processing the measured data-namely, the scattered field-with a reconstruction algorithm based on a model of scattering dealing with multiple scattering, leads to superresolution. In this paper we show that this concept, whose efficiency has been demonstrated for microscopy applications, can be applied for optical profilometry when high lateral resolutions are required. We stress in this case the importance of using a rigorous method for calculating the scattered field. In Section 2 we present the geometry of the problem. Section $\underline{3}$ is devoted to the description of the boundary integral formalism used for calculating rigorously the field scattered by rough surfaces. The numerical iterative algorithm used for reconstructing the surface profile from the scattered field is described in Section $\underline{4}$. In Section 5 we discuss, on the basis of numerical simulations, the effect of multiple scattering on the lateral resolution and the robustness of the inversion procedure.

\section{SURFACE SCATTERING}

For simplicity, we consider two-dimensional (2D) timeharmonic scattering from a perfectly conducting rough surface illuminated by TE-polarized electromagnetic beams (Fig. 1). In a Cartesian coordinates system $(x, y, z)$, the invariance direction is the $y$ axis and the surface is described by the profile $\Gamma: z=\eta(x)$, with a normal unit vector $\hat{\mathbf{n}}$ directed toward the air $z>\eta(x)$. At pulsation $\omega$, with a $\exp (-i \omega t)$ time dependence assumed and in the TE case, $\psi^{\text {inc }}\left(\mathbf{r}, \theta_{0}\right)$ is the $y$ component of the complex incident electric field at point $\mathbf{r}=$ $(x, z)$ of the incident beam centered on the illumination angle $\theta_{0} . \psi^{\text {inc }}$ writes as the sum of downward propagating plane waves:

$$
\begin{aligned}
\psi^{\text {inc }}\left(\mathbf{r}, \theta_{0}\right)= & \frac{g}{2 \sqrt{\pi}} \int_{\left|k_{x}\right|<k} \exp \left(i k_{x} x-i \sqrt{\left.k^{2}-k_{x}^{2} z\right)}\right. \\
& \times \exp \left[-\left(k_{x}-k \sin \theta_{0}\right)^{2} g^{2} / 4\right] \mathrm{d} k_{x},
\end{aligned}
$$




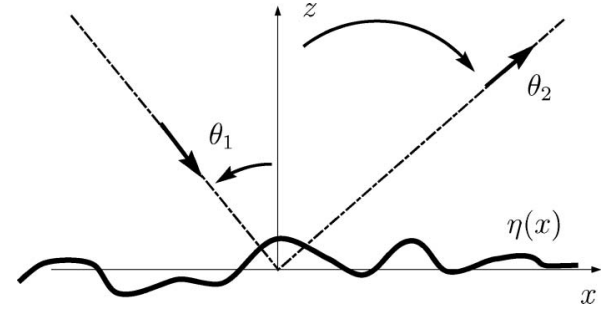

Fig. 1. Geometry of the surface scattering problem.

where $g$ is the tapering parameter [10] that sets the footprint size of the beam on the profile.

The total field $\psi=\psi^{\text {inc }}+\psi^{\text {sca }}$ is the sum of the incident and scattered field, and satisfies, in the TE case and for a onedimensional (1D) perfectly conducting surface, the Dirichlet boundary condition:

$$
(\mathbf{r} \in \Gamma \Leftrightarrow z=\eta(x)) \Rightarrow \psi\left(\mathbf{r}, \theta_{0}\right)=0 .
$$

In the far field along the direction of the wave vector,

$$
\mathbf{k}=k \frac{\mathbf{r}}{r}=(k \cos \theta, k \sin \theta), \quad k=\frac{\omega}{c},
$$

the scattered field writes in the air as a cylindrical wave:

$$
\psi^{\mathrm{sca}}\left(\mathbf{r}, \theta_{0}\right) \sim \frac{(1+i) e^{i k r}}{4 \sqrt{\pi k r}} s\left(\theta, \theta_{0}\right),
$$

whose complex amplitude is proportional to the so-called scattering amplitude $s\left(\theta, \theta_{0}\right)$, which depends on both the illumination angle $\theta_{0}$ and the detection angle $\theta$. Note that the optical intensity is classically defined as $I=|s|^{2}$.

The direct surface scattering problem corresponds to the calculus of the $s\left(\theta, \theta_{0}\right)$ for a given profile $z=\eta(x)$, while the inverse surface profiling is then stated as determining the surface $\eta(x)$ from the knowledge of the scattering amplitude $s\left(\theta, \theta_{0}\right)$.

\section{BOUNDARY INTEGRAL FORMALISM}

The boundary integral equation (BIE) method is the numerical solution of a rigorous wave scattering theory. It includes all multiple scattering with no simplifying assumption. It states that, provided the media are homogeneous, the whole problem can be solved on the surface. In the case detailed in Section 2 , the normal derivate of the electric field $\partial_{n} \psi=$ $\hat{\mathbf{n}} \cdot \operatorname{grad} \psi$ on $\Gamma$ is the unknown of the problem and satisfies the integral equation

$$
\mathbf{r} \in \Gamma, \int_{\Gamma} G\left(\mathbf{r}, \mathbf{r}^{\prime}\right) \partial_{n} \psi\left(\mathbf{r}^{\prime}, \theta_{0}\right) \mathrm{d} \mathbf{r}^{\prime}=-\psi^{\mathrm{inc}}\left(\mathbf{r}, \theta_{0}\right),
$$

with kernel $G\left(\mathbf{r}, \mathbf{r}^{\prime}\right)=-i H_{0}^{+}\left(k\left|\mathbf{r}-\mathbf{r}^{\prime}\right|\right) / 4$ as the $2 \mathrm{D}$ free space Green function, $H_{0}^{+}$as the first kind Hankel function of zero order, and $\mathrm{d} \mathbf{r}^{\prime}=\sqrt{1+\eta^{\prime}\left(x^{\prime}\right)^{2}} \mathrm{~d} x^{\prime}$ as the element of profile length.

Once Eq. (5) is solved for a given illumination angle $\theta_{0}$, the scattering amplitude reads

$$
s\left(\theta, \theta_{0}\right)=-\int_{\Gamma} \partial_{n} \psi\left(\mathbf{r}, \theta_{0}\right) \exp (-i \mathbf{k} \cdot \mathbf{r}) \mathrm{d} \mathbf{r},
$$

at any detection angle $\theta$ in the air.
The discretization and numerical solving of the integral in Eq. (5) is detailed in [11]. The BIE constitutes a rigorous direct model, but it cannot be directly inversed.

If one assumes both single and paraxial scattering, one can easily show that the scattering amplitude is linearly related to the Fourier transform $\tilde{f}(k)$ of some function $f(x)$ :

$$
s\left(\theta, \theta_{0}\right)=\tilde{f}\left(k \sin \theta-k \sin \theta_{0}\right),
$$

from which the profile can be retrieved directly [12] by using

$$
\eta(x)=\frac{\lambda}{4 \pi} \arg f(x)
$$

This simple inversion technique is used in most holographic or phase microscopy experiments [13]. The transverse resolution is then easily estimated from the spatial frequency span of $\tilde{f}$ that is accessible with the given illumination and detection angles. Note that, in [14], single scattering inversions of $2 \mathrm{D}$ surfaces are performed without the paraxial approximation, but with somehow equivalent results: the main and limiting assumption is single scattering.

\section{INVERSE SCATTERING}

Assume that a surface characterized by its variation of the height $\eta(x)$ is successively illuminated by $l=1, \ldots, L$ different beams and, for each illumination $l$, the scattered far field is measured for $m=1, \ldots, M$ different angles of detection. If single scattering is not assumed, the link between the profile and the scattered field is nonlinear. The inversion problem is then generally recast as an optimization problem. In this section we describe the Newton-Kantorovitch (NK) method $[\underline{15}, \underline{16}]$ to solve the nonlinear equation relating the data set to the surface $\Gamma$. For the sake of simplicity, symbolic notation is introduced:

$$
s^{\mathrm{mes}}=\mathbf{F} \eta,
$$

where $s^{\text {mes }}$ denotes the measured scattered far field. In practice, it is a complex valued array of size $(L \times M)$ with $L$ being the number of illuminations and $M$ the number of detection angles. F represents the symbolic link between the measured scattered field and the height of the surface under test $\eta$. It involves coupled integral representations of fields reported in Eqs. (5) and (6).

The NK method iteratively builds up the solution of Eq. (9) by successively solving the forward problem and a local linear inverse problem. At each iteration step $n$, an estimate of the surface profile function is given by

$$
\eta_{n}=\eta_{n-1}+\delta \eta_{n}
$$

where $\delta \eta_{n}$ is an update correction that is obtained by solving in the least squares sense the linearized forward problem:

$$
\mathbf{D} \delta \eta_{n}=\delta s=\left(s^{\text {mes }}-s_{n-1}\right),
$$

with $s_{n-1}$ as the scattered far field associated to the best available estimation of the surface $\eta_{n-1}$ computed thanks to Eqs. (5) and (6), while $\mathbf{D}$ is the Fréchet derivative of the nonlinear operator $\mathbf{F}$ relating a small variation of scattered far field $\delta s$ to a small variation of the object function $\delta \eta$. The derivation of $\mathbf{D}$ is based on the reciprocity theorem and full details of this 
derivation can be found in [17]. To sum it up briefly, consider two angles $\theta_{1}$ and $\theta_{2}$; the variation of the scattered far field $\delta s$ is linked to the variation of the profile $\delta \eta$ through the following relation [17]:

$$
\delta s\left(\theta_{2}, \theta_{1}\right)=-\int_{\Gamma} \partial_{n} \psi\left(\mathbf{r}, \theta_{1}\right) \partial_{n} \psi\left(\mathbf{r},-\theta_{2}\right) \delta \eta(\mathbf{r}) \mathrm{d} \mathbf{r}
$$

where $\partial_{n} \psi\left(\mathbf{r}, \theta_{1}\right)$ is the current that would be present at the surface $\eta_{n-1}$ when illuminated by a beam of angle of incidence $\theta_{1}$, while $\partial_{n} \psi\left(\mathbf{r}, \theta_{1}\right)$ is the current that would be present at the same surface but with an illuminating beam of angle of incidence $-\theta_{2}$. The key issue of the evaluation of $\mathbf{D}$ is that it requires only the computation of an adjoint forward problem where the current distribution at the surface is determined for all illuminating angles $-\theta_{i}$. Unfortunately, the problem of finding the solution of Eq. (11) is ill-posed and needs regularization. We use a zeroth-order standard Tikhonov regularization [18]:

$$
\left[\mathbf{D}^{\dagger} \mathbf{D}+\mu^{2} \mathbf{I}\right] \delta \eta_{n}=\mathbf{D}^{\dagger}\left(s^{\text {mes }}-s_{n-1}\right),
$$

where $\mu^{2}$ is the regularization parameter that does not vary during the iterative process, $\mathbf{I}$ is the identity matrix, and the $\dagger$ symbol stands for the transposed complex conjugate. In practice, this parameter is chosen by trial and error. For the numerical experiments reported in this article, $\mu^{2}$ takes a value of $10^{6}$ or $10^{8}$ and seems to not depend on the presence of noise in data.

\section{NUMERICAL RESULTS}

In the following, the wavelength is set to $\lambda=633 \mathrm{~nm}$, with both incidences and scattering angles regularly sampled be-

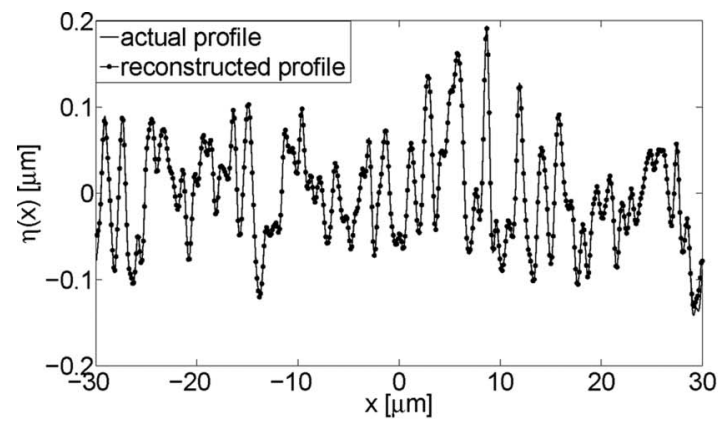

(a)

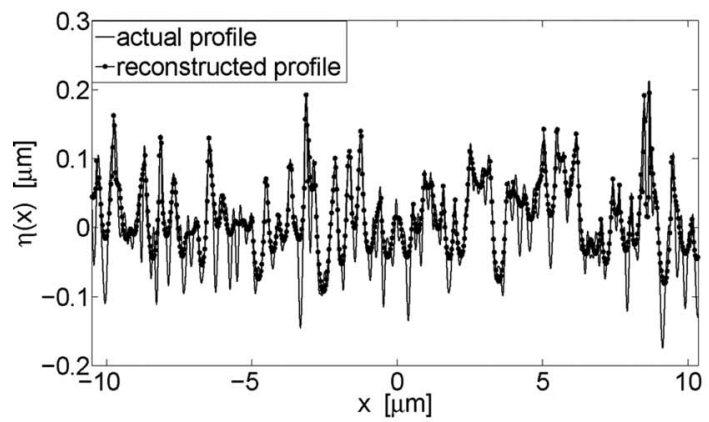

(c) tween $-45^{\circ}$ and $+45^{\circ}$ with a $1^{\circ}$ step $(L=M=91)$. The numerical aperture is thus $\mathrm{NA}=0.71$ and the single scattering analysis states [19] that the minimum distance between two resolved points is $\lambda /(4 \mathrm{NA})=223 \mathrm{~nm}$.

All data are generated thanks to the rigorous direct model where the integral equation [Eq. (5)] is cast into a matrixvector form according to the now classical procedure detailed in [11]. Note that different mesh sizes are used for generating the data and performing the inversion.

We first consider rough surfaces of $60 \mu \mathrm{m}$ length with two different Gaussian correlations $\ell=500 \mathrm{~nm}$ and $\ell=100 \mathrm{~nm}$ and the same rms height of $60 \mathrm{~nm}$ (Fig. 2). Each profile is sampled with $N=4096$ points to compute the scattered field that corresponds to the data. For inversion, the estimated profile is also $60 \mu \mathrm{m}$ long but counts only $N=2048$ points.

For the smooth surface, $\ell=500 \mathrm{~nm}$, both the NK and linear inversion methods provide good reconstruction of the profile [Figs. 2(a) and 2(b)]. The axial accuracy of the reconstruction given by the $\mathrm{NK}$ algorithm is spectacular (within the nanometer). For the rougher surface, $\ell=100 \mathrm{~nm}$, the reconstruction given by the NK algorithm is still accurate, while that obtained under the Fraunhofer approximation clearly misses the high frequencies of the actual profile [Figs. 2(c) and 2(d)]. This example stresses the importance of a rigorous modeling of the light-surface interaction when surfaces with subwavelength transverse features are to be studied. Note that the edges of the profile are poorly reconstructed by the Fraunhofer method, as can be seen in Fig. 2(b). This is a numerical artifact due to our implementation of the Fraunhofer method.

Following the analysis given in [9], we have also checked the ability of the NK algorithm to provide, in the multiple scattering regime, a transverse resolution better than that classically expected. We consider a surface profile made of two

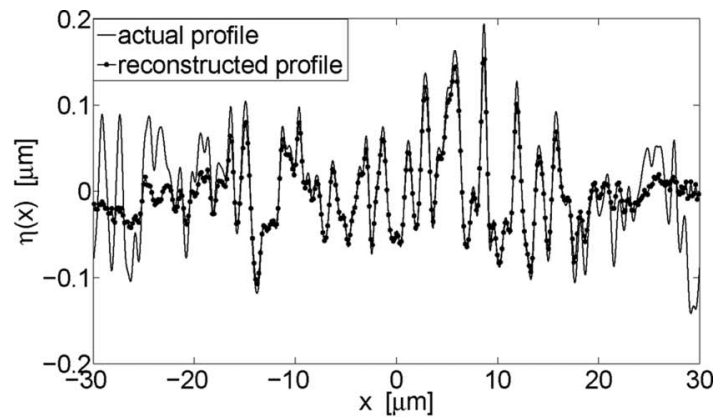

(b)

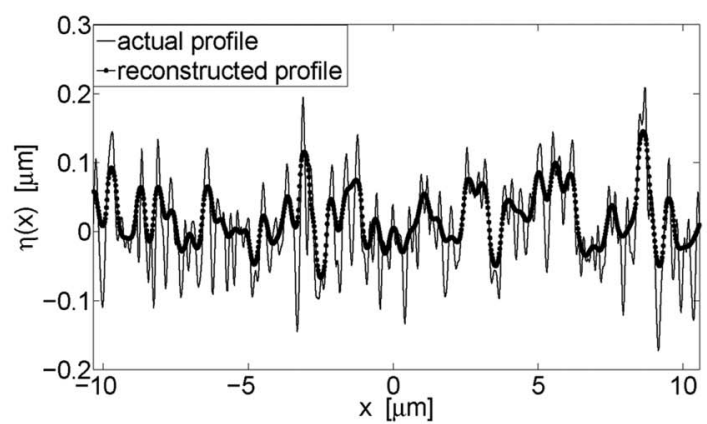

(d)

Fig. 2. Reconstruction of $60 \mu \mathrm{m}$ long rough surfaces with Gaussian correlation with rms height of $60 \mathrm{~nm}$ and different values of the correlation length $\ell$. (a) and (b) $\ell=500 \mathrm{~nm}$, and (c) and (d) $\ell=100 \mathrm{~nm}$. (a) and (c) Reconstructed profiles using NK, while (b) and (d) are reconstructed profiles using the Fraunhofer approximation. In (c) and (d), only the $20 \mu \mathrm{m}$ central part is reported. 


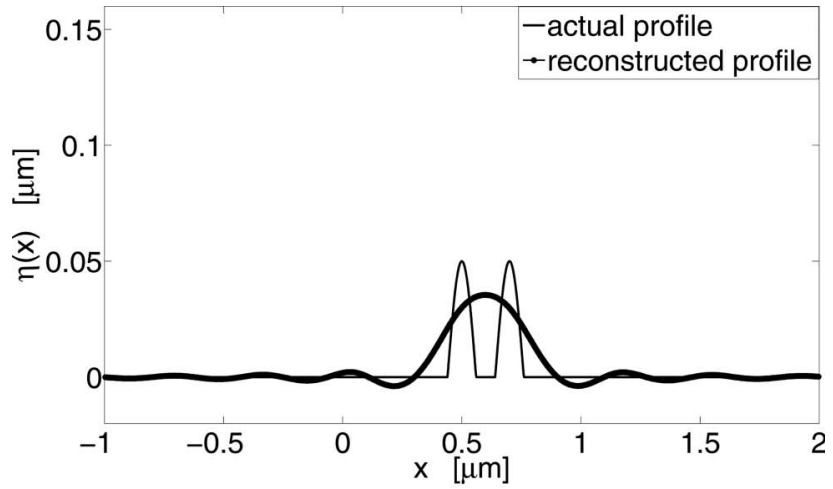

(a)

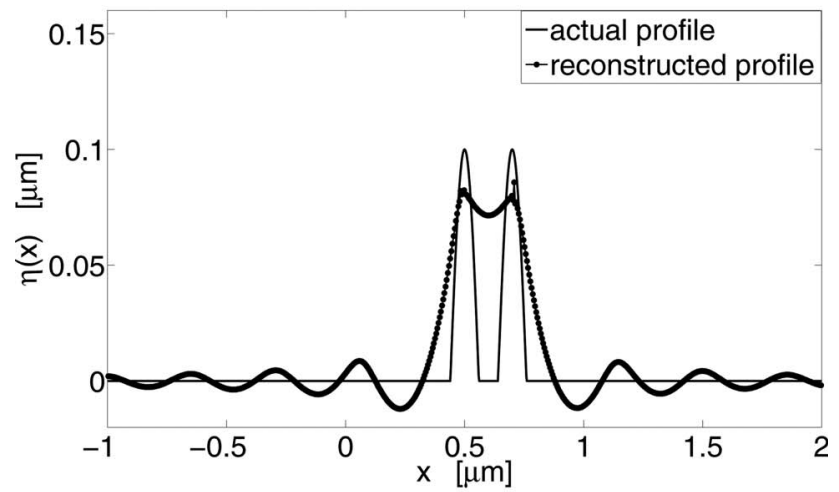

(b)

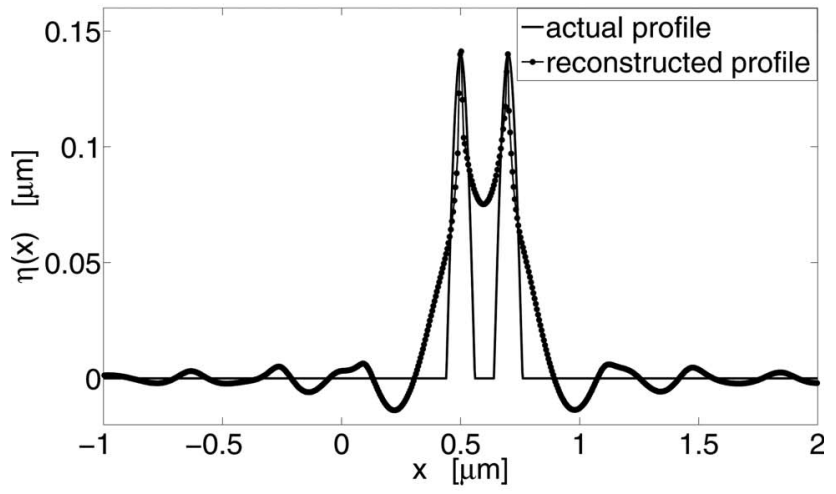

(c)

Fig. 3. Reconstruction at $\lambda=633 \mathrm{~nm}$ using the NK algorithm of a surface constituted of two bumps of $w=80 \mathrm{~nm}$ width and separated by a fixed interdistance $d=200 \mathrm{~nm}$, but with various values of the height $h$. (a) $h=50 \mathrm{~nm}$; (b) $h=100 \mathrm{~nm}$; (c) $h=140 \mathrm{~nm}$.

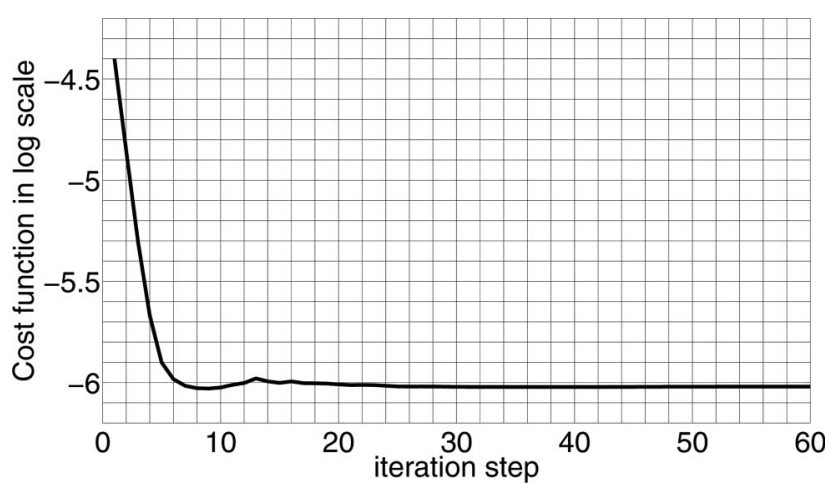

Fig. 4. Evolution of the cost function against the iteration step for the reconstruction that corresponds to Fig. $3(\mathrm{c})$. identical bumps of width $w=80 \mathrm{~nm}$ and interdistance $200 \mathrm{~nm}$, which is below the resolution given by the single scattering analysis, $223 \mathrm{~nm}$. The height of the bumps is increased from 50 to $180 \mathrm{~nm}$. In all these experiments, the beam parameter is set to $g=2.5 \mu \mathrm{m}$. We plot in Fig. 3 the reconstructed profiles obtained with the NK algorithm. When the bumps' height $h=50 \mathrm{~nm}$, is much smaller than the incident wavelength, only single scattering is significant. The linear inversion method and the NK algorithm give the same result, and the bumps cannot be distinguished. When the bumps' height increases, multiple scattering becomes more important and the NK algorithm is able to distinguish the two bumps more and more distinctly. This numerical experiment clearly shows that the presence of multiple scattering can be an advantage for a nonlinear reconstruction procedure.

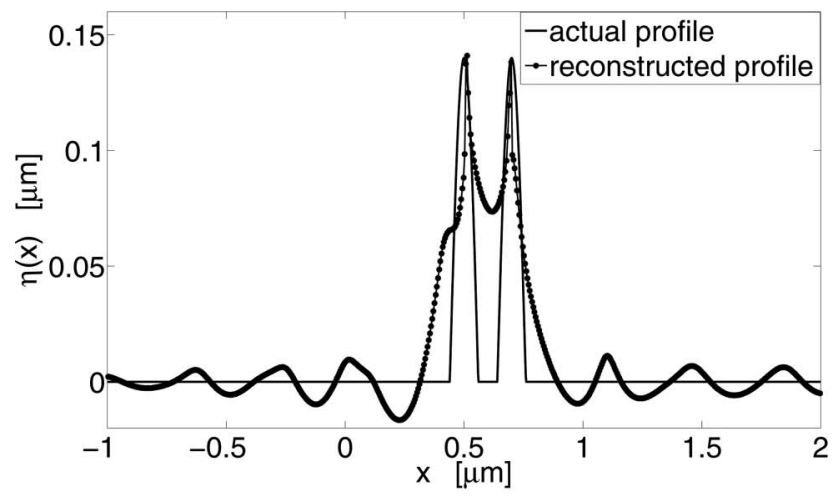

(a)

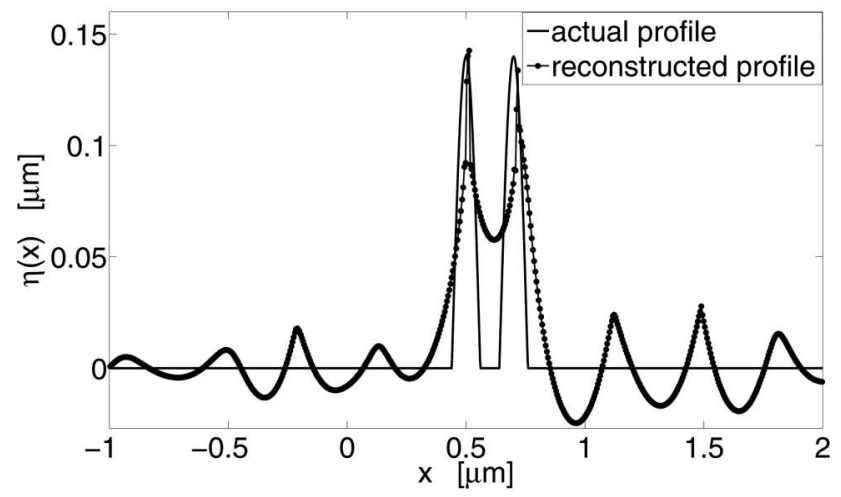

(b)

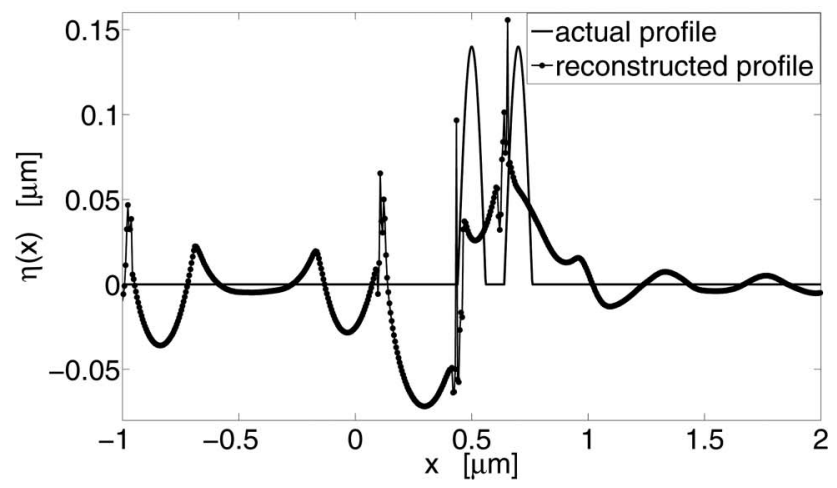

(c)

Fig. 5. Reconstruction, at $\lambda=633 \mathrm{~nm}$ and using the NK algorithm, of a surface constituted of two bumps of $w=80 \mathrm{~nm}$ width and height $h=140 \mathrm{~nm}$, separated by a fixed interdistance $d=200 \mathrm{~nm}$ from noisy data. The SNRs are (a) 50, (b) 20, and (c) 10. 
We add also, in the example of the two bumps that corresponds to Fig. 3(c), the evolution of the cost function with respect to iteration steps (Fig. 4). One can observe that this cost function decreases and reaches a "plateau." The minimum value of the cost function is reached at iteration step 9. However, this may be misleading, because the reconstruction at iteration step 60 appears to be more accurate than the one at step 9 .

To check the robustness of the NK inversion method in the presence of noise, we have added a complex white noise to the data. The profile is the same as for Fig. 3(c), and we have considered a noise amplitude proportional to the maximum value of the scattering amplitude, with three different signal-to-noise ratios (SNRs), namely 50, 20, and 10. It appears in Fig. $\underline{5}$ that the performances of the NK are only slightly deteriorated for the two first SNRs.

\section{CONCLUSION}

In conclusion, we believe that tomographic diffractive microscopy combined with a rigorous model of the light-surface interaction is an interesting tool for profilometry applications. This approach can deal with surfaces presenting strong slopes that cannot be imaged with classical optical far-field profilometers. The occurrence of multiple scattering can be an advantage as it can lead to a subwavelength lateral resolution that can be a crucial advantage. The results presented in this paper will encourage an experimental validation with a tomographic diffraction setup. The actual theory can be applied to data on $1 \mathrm{D}$ roughness, such as grooves or lines. To work on $2 \mathrm{D}$ surfaces, the method has to be extended. It is only a numerical methods matter, since both the boundary integral equations and the Fréchet derivative, generalizations of Eqs. (5) and (12), have already been published.

\section{REFERENCES}

1. D. Huang, E. A. Swanson, C. P. Lin, J. S. Shuman, W. G. Stinson, W. Chang, M. R. Hee, T. Flotte, K. Gregory, C. A. Puliafito, and J. G. Fujimoto, “Optical coherence tomography," Science 254, 1178-1181 (1991).

2. C. J. Brakenhoff, P. Blom, and P. Barends, "Confocal scanning light microscopy with high aperture immersion lenses," J. Microsc. 117, 219-232 (1979).
3. T. Wilson and C. J. R. Sheppard, Theory and Practice of Scanning Optical Microscopy (Academic, 1984).

4. G. Q. Xiao, T. R. Corle, and G. S. Kino, "Real time confocal scanning optical microscope,” Appl. Phys. Lett. 53, 716-718 (1988).

5. H. J. Tiziani and H. M. Uhde, "Three-dimensional image sensing by chromatic confocal microscopy," Appl. Opt. 33, 1838-1843 (1994).

6. V. Lauer, "New approach to optical diffraction tomography yielding a vector equation of diffraction tomography and a novel tomographic microscope,” J. Microsc. 205, 165-176 (2002).

7. O. Haeberlé, K. Belkebir, H. Giovannini, and A. Sentenac, "Tomographic diffractive microscopy: basics, techniques and perspectives,” J. Mod. Opt. 57, 686-699 (2010).

8. G. Maire, F. Drsek, J. Girard, H. Giovannini, A. Talneau, D. Konan, K. Belkebir, P. C. Chaumet, and A. Sentenac, "Experimental demonstration of quantitative imaging beyond Abbe's limit with optical diffraction tomography," Phys. Rev. Lett. 102, 213905 (2009).

9. K. Belkebir, P. C. Chaumet, and A. Sentenac, "Influence of multiple scattering on three-dimensional imaging with optical diffraction tomography," J. Opt. Soc. Am. A 23, 586-595 (2006).

10. E. Thorsos, "The validity of the Kirchhoff approximation for rough surface scattering using a Gaussian roughness spectrum," J. Acoust. Soc. Am. 83, 78-92 (1988).

11. L. Tsang, J. A. Kong, K. H. Ding, and C. O. Ao, Scattering of Electromagnetic Waves: Numerical Simulations, Wiley Series in Remote Sensing (Wiley-Interscience, 2001).

12. J. Goodman, Introduction to Fourier Optics (Roberts, 2005).

13. O. Haeberlé, K. Belkebir, H. Giovaninni, and A. Sentenac, "Tomographic diffractive microscopy: basics, techniques and perspectives,” J. Mod. Opt. 57, 686-699 (2010).

14. R. Wombell and J. DeSanto, "Reconstruction of rough-surface profiles with the Kirchhoff approximation," J. Opt. Soc. Am. A 8, 1892-1897 (1991).

15. A. Roger, "Newton-Kantorovitch algorithm applied to an electromagnetic inverse problem," IEEE Trans. Antennas Propag. 29 232-238 (1981).

16. K. Belkebir, R. E. Kleinman, and C. Pichot, "Microwave imaging -location and shape reconstruction from multifrequency scattering data," IEEE Trans. Microwave Theory Tech. 45, 469-476 (1997).

17. A. Roger, "Reciprocity theorem applied to the computation of functional derivatives of the scattering matrix," Electromagnetics 2, 69-83 (1982).

18. A. N. Tikhonov and V. A. Arsenin, Solutions of Ill-Posed Problems (Wiley, 1977).

19. O. Haeberlé, A. Sentenac, and H. Giovannini, "An introduction to diffractive tomographic microscopy," in Modern Research and Educational Topics in Microscopy, A. M. Vilas and J. D. Alvarez, eds. (2007), Vol. 2. 